Genome Insight Plant Genetics

\title{
The complete plastid genome of Cotinus coggygria and phylogenetic analysis of the Anacardiaceae
}

\author{
Lingfeng $\mathrm{Xu}^{1 *}$, Nong Zhou ${ }^{1 *}$, Shunxin Zhao ${ }^{1}$, Jingling $\mathrm{Li}^{2}$ (D), Xiaoying $\mathrm{Pei}^{2},{\mathrm{Jie} \mathrm{Yu}^{2} \text {, Dongqin Guo }}^{1}$ \\ ${ }^{I}$ Chongqing Three Gorges University, Chongqing, College of Biology and Food Engineering, \\ Chongqing Engineering Laboratory of Green Planting and Deep Processing of Genuine Medicinal \\ Materials in the Three Gorges Reservoir Region, China \\ ${ }^{2}$ Southwest University, College of Horticulture and Landscape Architecture, Chongqing, China
}

\begin{abstract}
Cotinus coggygria Scop. (Anacardiaceae) is an important ornamental tree with beautiful characteristics that is grown in China. In this study, the complete plastid genome of C. coggygria was sequenced and assembled. This genome was $158,843 \mathrm{bp}$ in size and presented a typical tetrad structure, consisting of a large single-copy region (87,121 bp), a pair of inverted repeat regions $(26,829 \mathrm{bp})$, and a small single-copy region (18,064 bp). A total of 134 genes were annotated, including 88 protein-coding genes, 38 tRNA genes, and 8 rRNA genes. We observed a deletion that caused the loss of the $r p / 32$ gene, and a small expansion of IR regions resulted in the $t r n \mathrm{H}$ gene accessing IR regions; two copies were obtained. Phylogenetic analysis showed that C. coggygria was most closely related to Pistacia, with $100 \%$ bootstrap support within Anacardiaceae. In this study, we report the plastid genome of Cotinus species for the first time, which provides insight into the evolution of the plastid genome in Anacardiaceae and promotes the understanding of Cotinus plants.
\end{abstract}

Keywords: Plastid genome; genes; genetic resource; structure variation; phylogenetic analysis.

Received: January 7, 2021; Accepted: June 2, 2021.

Cotinus is a small genus in Anacardiaceae and is mainly distributed in southern Europe, eastern Asia and temperate regions of North America (Matić et al., 2016). Cotinus coggygria Scop., commonly known as "smoke tree", is usually considered either a large shrub or a small tree. This plant is cultivated in large urban parks, mountain scenic areas and gardens due to its brightly colored leaves and hardiness in barren soil (Miao et al., 2017). In late autumn, the leaves of $C$. coggygria are brightly colored and beautiful. In northern China, due to the cold climates, garden tree species are relatively monotonous and lack color. C. coggygria is the first choice for afforestation in northern landscapes or mountainous areas in China. The Fragrant Hills Park in Beijing has planted a large number of smoke trees, known as "fragrant hills red leaves".

In this study, we sequenced and characterized the complete plastid genome of Cotinus coggygria and carried out a comparative study of Anacardiaceae plants. Our main analyses are as follows: 1) we sequenced and assembled the complete plastome sequences of C. coggygria for the first time; 2) the structural characteristics of $C$. coggygria plastomes were analyzed; 3) the boundaries of IR regions of plastomes were analyzed and described; and 4) the phylogenetic relationships of C. coggygria were inferred based on the complete plastome sequences. The results obtained here will provide a reference for the phylogenetic inference of Cotinus and studies on the evolution of plastomes in Anacardiaceae.

Send correspondence to Dongqin Guo. Chongqing Three Gorges University, Chongqing, College of Biology and Food Engineering, Chongqing Engineering Laboratory of Green Planting and Deep Processing of Genuine Medicinal Materials in the Three Gorges Reservoir Region, 404120, China. E-mail: guodongqin1997@ 163.com. ${ }^{*}$ These authors contributed equally.
Fresh leaves of C. coggygria were collected from Mount Jinyun (geospatial coordinates: N29.842889, E106.394527), Chongqing, China. The samples were deposited in the Herbarium of Southwest University, Chongqing, with the accession number SWU-CQ2. Total genomic DNA was extracted using the CTAB method (Arseneau et al., 2017). The total DNA was ultrasonically fragmented. A DNA library with an insert size of $350 \mathrm{bp}$ was constructed using the NEBNext ${ }^{\circledR}$ library building kit (Emerman et al., 2017) and was sequenced using the HiSeq Xten PE150 sequencing platform. Sequencing produced a total of $5.9 \mathrm{~Gb}$ raw data with $21,318,311$ raw reads. Clean data were obtained using Trimmomatic (Bolger et al., 2014): we removed the low-quality sequences with more than $5 \%$ bases being " $\mathrm{N}$ ", and more than $50 \%$ of the total bases had a quality value of $\mathrm{Q}<19$. Ultimately, 21,200,559 clean reads were obtained after trimming.

De novo genome assembly from the clean data was accomplished utilizing NOVOPlasty v2.7.2 (Dierckxsens et al., 2017), with a k-mer length of $39 \mathrm{bp}$ and a sequence fragment of the $r b c \mathrm{~L}$ gene from maize as the seed sequence. A total of 286,982 reads were used in the final plastid assembly, and a circular genome was obtained. The average sequence coverage was 646. Bowtie2 (v2. 0.1) (Langmead et al., 2009) was used to ensure the correctness of the assembly by mapping all clean reads to the assembled genome sequence. The CPGAVAS2 (Shi et al., 2019) program was used to annotate the genome with the reference genome (Pistacia chinensis, GenBank: NC_046786.1). Apollo (Misra and Harris, 2006) was used to manually edit any annotations with problems. The genome maps were drawn in OGDRAW (Greiner et al., 2019). The genome sequence and raw sequencing data have been deposited in GenBank with the accession numbers MT876478 and SRR13076877. 
The complete plastome sequence of $C$. coggygria is $158,843 \mathrm{bp}$ in size and presents a typical tetrad structure, consisting of a large single-copy (LSC) region, a pair of inverted repeat (IR) regions, and a small single-copy (SSC) region, with lengths of $87,121 \mathrm{bp}, 26,829 \mathrm{bp}$, and $18,064 \mathrm{bp}$, respectively. This structure is similar to those of other plants of Anacardiaceae. A total of 134 genes were annotated in the plastome of C. coggygria (Table S1, Figure 1), including 88 protein-coding genes, 38 tRNA genes, and 8 rRNA genes. We noted that the rpl32 gene was not annotated in this plastome. The $r p l 32$ gene encodes a ribosomal protein and is the structural constituent of the $50 \mathrm{~S}$ ribosome, which is considered to be involved in the biological process of translation (Weglöhner and Subramanian, 1993; Gaudet et al., 2011). We retrieved the sequence of $r p l 32$ from a closely related species (Pistacia chinensis) and performed a Bowtie 2 search on the complete raw data. Only 5 reads were partially mapped, and the sequence source of the unmapped part could not be identified after BLAST (Altschul et al., 1990) retrieval. We found no evidence to support transfer to the nucleus; therefore, we hypothesized that the deletion caused the species to completely lose the gene.

Simple sequence repeats (SSRs) were identified using the online website MISA(Beier et al., 2017), including mono-, di-, tri-, tetra-, penta-, and hexanucleotides with minimum numbers of $10,5,4,3,3$, and 3 , respectively. We detected 88 SSRs in the plastomes of C. coggygria. (Table S2). Most SSRs are mononucleotide homopolymers, particularly polyA (34) and polyT (35), which account for $88.75 \%$ of the total. In addition, there were 4 dinucleotides, 6 trinucleotides and 7 tetranucleotides.

The IRscope program (Amiryousefi et al., 2018) was used to visualize IR boundaries. The IR boundaries of $C$.

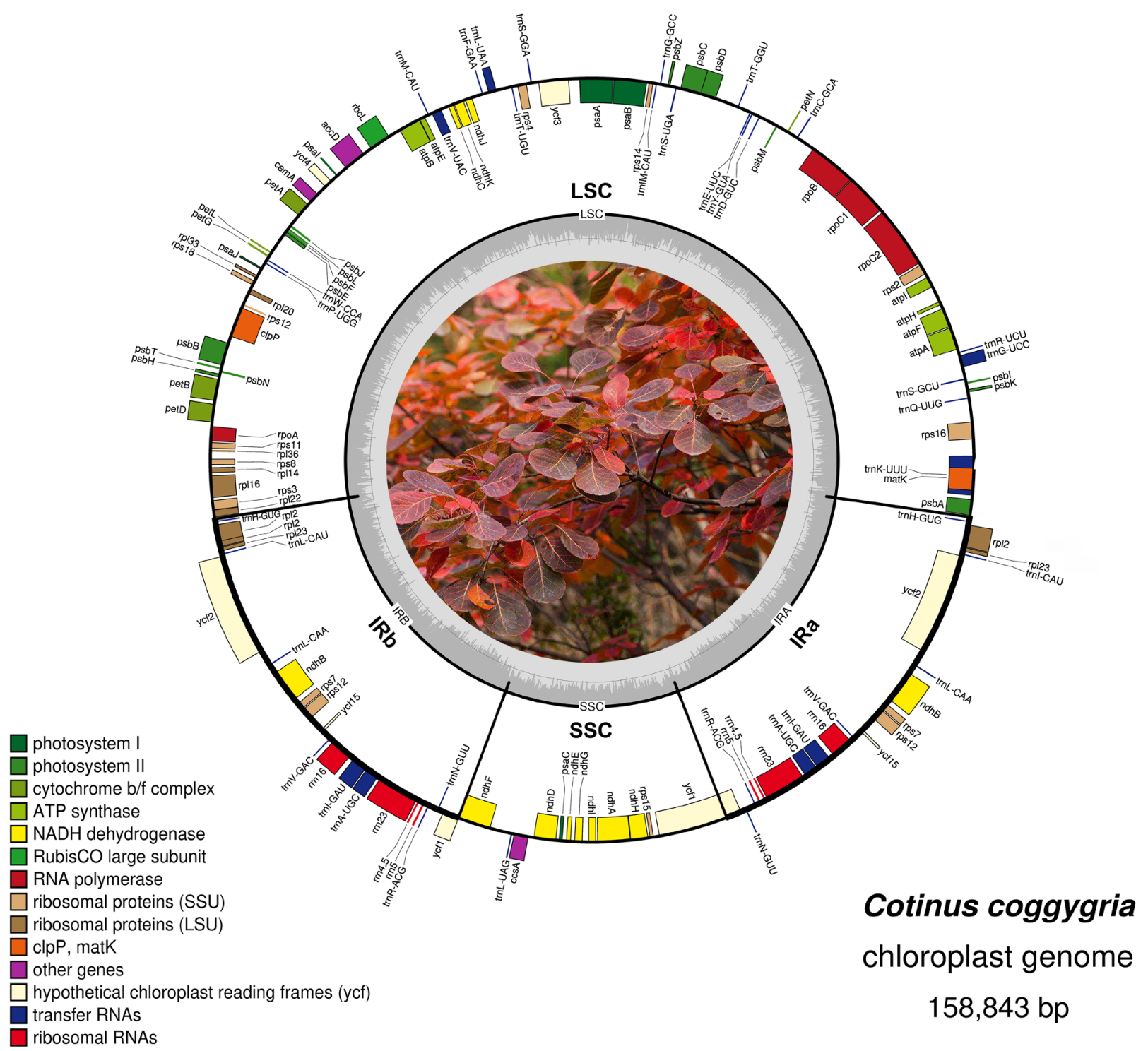

Figure 1 - Graphic representation of features identified in the cp genomes of C. coggygria by using OGDRAW. The plastome has a conservative quartile structure composed of a LSC region, a SSC region and a pair of IR regions. The genes outside the circle are transcribed in counter clockwise direction, and the genes inside the circle are transcribed in clockwise direction. Different colors in genes represent different functions. The dark gray and light gray areas of the inner circle represent the GC content to AT content of the genome, respectively. 
coggygria and seven other taxa from Anacardiaceae were analyzed, and the results are shown in Figure 2. In the SSC/ IRa boundary, the $y c f 1$ gene spans this boundary and is mostly located in the SSC region, and it overlaps with IRa by 1097$1104 \mathrm{bp}$. Spondias bahiensis is an exception, and the overlap is $1401 \mathrm{bp}$. At the IRb/SSC boundary, $y c f 1$ pseudogenes are produced by inverted repeats. Additionally, the $n d h \mathrm{~F}$ gene crosses the IRb/SSC boundary (except for Sclerocarya birrea) and forms a partial overlapping region with the $y c f 1$ pseudogenes. In general, there are no significant differences in SSC/IR boundaries.

At the LSC/IR boundary, we observed changes in the position of the rps 19 gene in different taxa. We found that rps 19 genes are completely located in the IR region in Spondias and Sclerocarya species; as a result, there are two complete copies in their genomes. In addition, the rps 19 gene spans the LSC/IRb boundary and is partially duplicated in the IRa regions in Anacardium and Mangifera species. However, in Toxicodendron, Rhus and Pistacia species, we observed that the rps 19 genes are located in the LSC region and are annotated as pseudogenes. Moreover, we observed a small expansion of IR regions in C. coggygria that resulted in the capture of the $t r n \mathrm{H}$ and rps 19 genes. The latter gene, rps 19 in C. coggygria, is also a fragment and is annotated as a pseudogene. The rps 19 gene encodes a ribosomal protein that is the structural constituent of the 40S ribosome, which is considered to be involved in the biological processes of translation and ribosomal small subunit assembly (Sánchez et al., 1996; Gaudet et al., 2011). Previously, rps 19 was also observed to be a pseudogene in Gentiana species (Sun et al., 2018).

To evaluate the divergence of plastomes among Anacardiaceae species, six plastomes (C. coggygria, $P$. chinensis, $R$. potaninii, T. sylvestre, S. birrea and S. bahiensis) were compared using shuffle-LAGAN mode in mVISTA (Brudno et al., 2003; Frazer et al., 2004) to identify interspecific variations. The reference annotation is $P$. chinensis. In addition, $M$. indica and $A$. occidentale were excluded from the analysis because it has been previously reported that their plastomes contain specific inversions in the LSC region or that there is a migration of DNA sequences from mitochondria in IR regions (Rabah et al., 2017). Therefore, they will not be discussed here. The IR regions are highly conserved compared to the SSC and LSC regions (Figure 3). Most of the regions with large divergence are observed in the intergenic regions, including $t r n \mathrm{H}-p s b \mathrm{~A}, p s b \mathrm{M}-t r n \mathrm{D}, t r n \mathrm{~T}-p s b \mathrm{D}, t r n \mathrm{~T}-t r n \mathrm{~L}, y c f 4-c e m \mathrm{~A}$, $p s b \mathrm{~F}-p e t \mathrm{~L}$ and $n d h \mathrm{~F}-r p l 32-t r n \mathrm{~L}$. In particular, a deletion is present between the $n d h \mathrm{~F}$ and $\operatorname{trn} \mathrm{L}$ genes and results in the complete loss of the rpl32 gene in C. coggygria. Among the protein-coding genes, rpoC2, rps 19 and $y c f 1$ show high sequence differences.

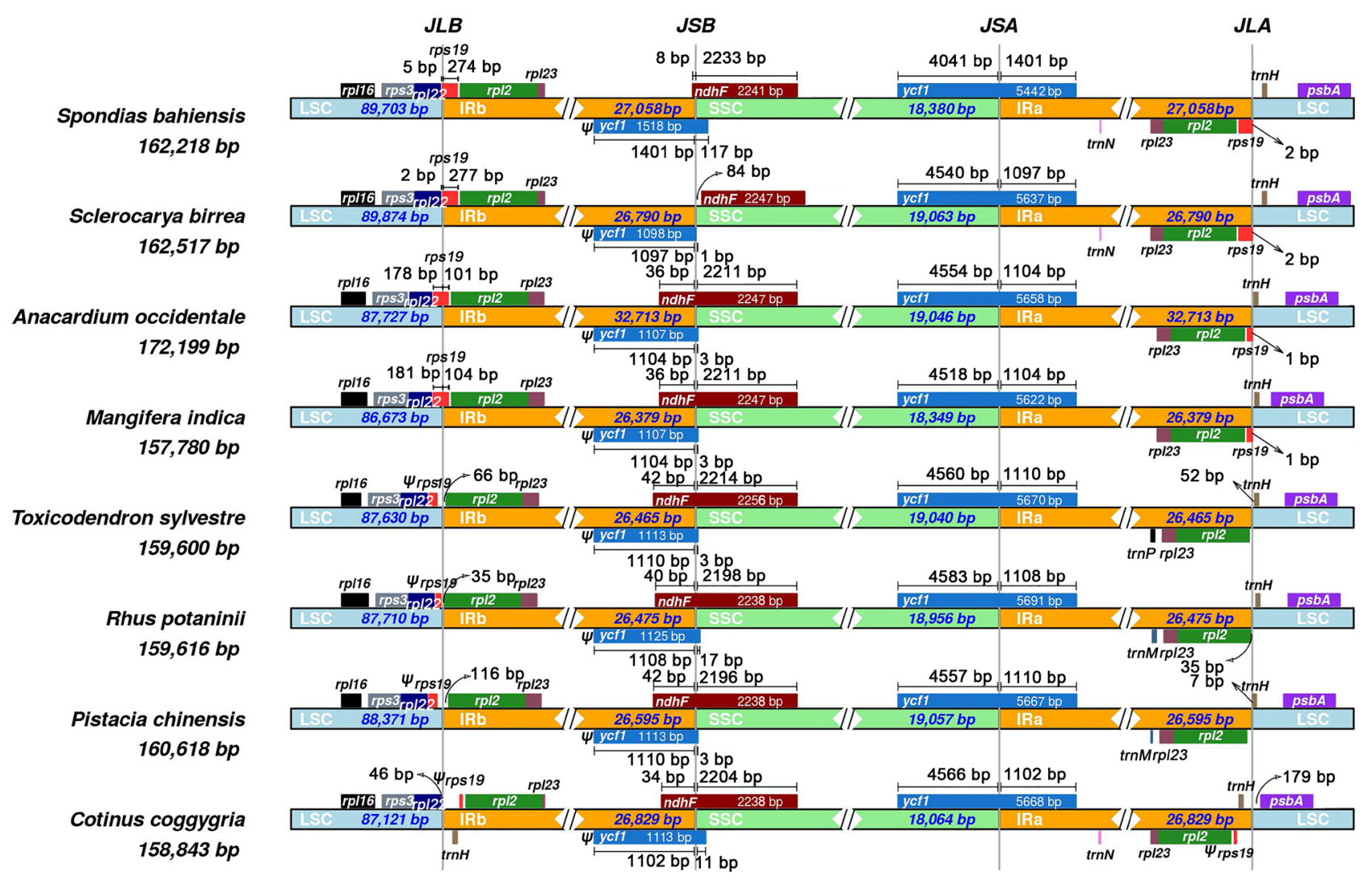

Figure 2 - Comparison of the borders among LSC, SSC, and IR regions of eight analyzed species. The genes around the borders are shown above or below the mainline. The JLB, JSB, JSA, and JLA represent junction sites of LSC/IRb, IRb/SSC, SSC/IRa, and IRa/LSC, respectively. 


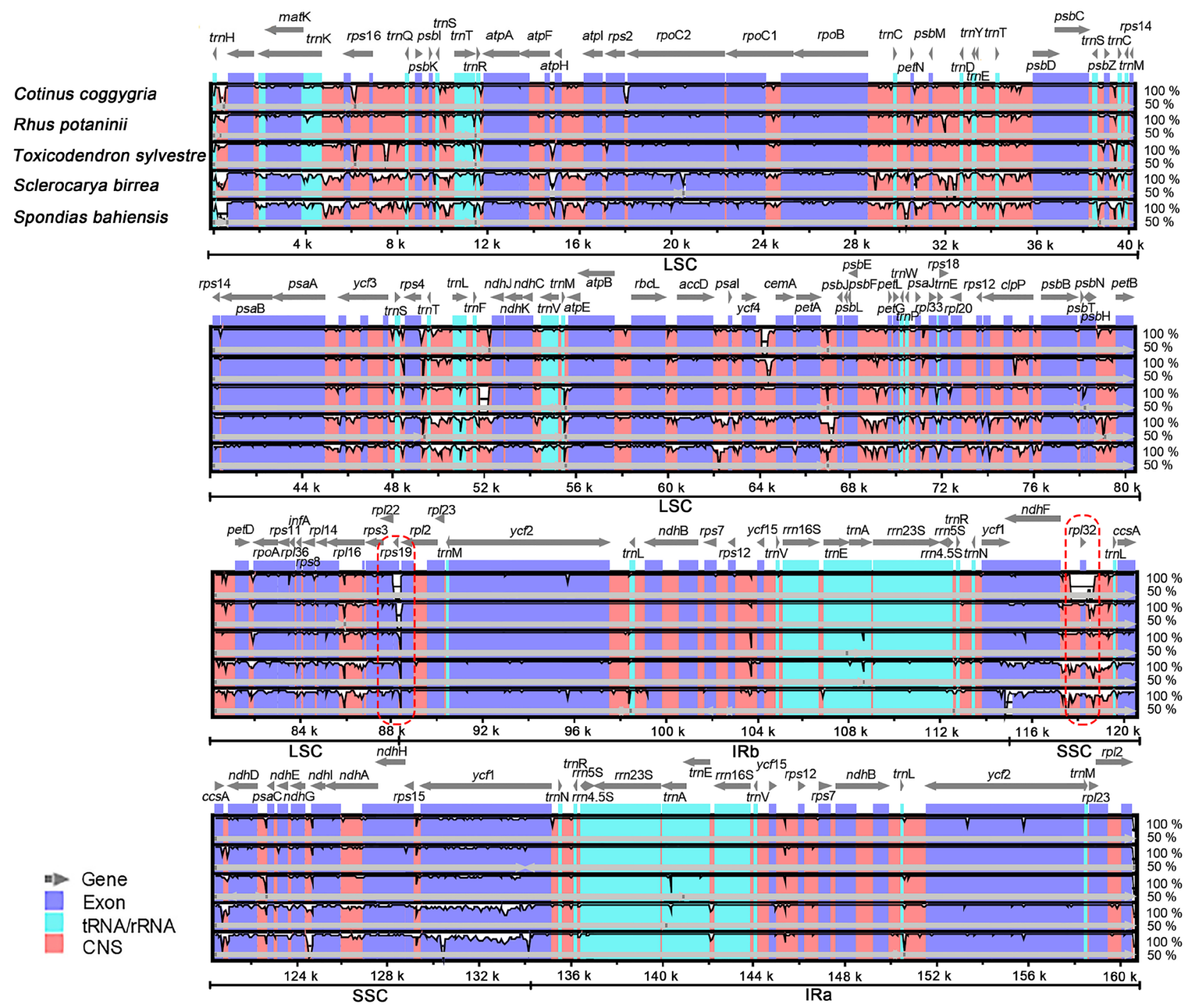

Figure 3 - Comparison of the cp genomes in six Anacardiaceae species by using mVISTA. The genes were represented as gray arrows on the top of the alignment. Different regions are labeled with different colors. The pink regions are "Conserved Non-Coding Sequences" (CNS), the dark blue regions are exons, and the light-blue regions are tRNA or rRNA. 50\% and 100\% refer to the similarity among sequences. Gray arrows above the aligned sequences represent genes and their orientation. In particular, two genes ( $r p s 19$ and rpl32) are circled in red, as they are quite different in these taxa. The deletion of a large fragments in C. coggygria led to the loss of rpl32 gene.

To determine the phylogenetic position of C. coggygria in Anacardiaceae, we reconstructed maximum likelihood (ML) trees based on the plastome sequences. The plastid genome sequences of 16 species belonging to the family Anacardiaceae were downloaded from GenBank. Two Burseraceae species, Boswellia sacra and Canarium album, were used as outgroups. A detailed list of all plastid genomes analyzed in this paper is provided in Table S3. The complete plastid genome sequences were aligned using MAFFT online version 7.471 (Rozewicki et al., 2019). To avoid negative effects of sequence inversion and migration sequences from mitochondria on phylogenetic reconstruction, a total of 1,477 highly conservative blocks were identified using Gblock (Castresana, 2000; Talavera and Castresana, 2007) with the default setting. The Gblock alignment (138,527 bp) accounted for $71 \%$ of the original alignment (194,522 bp). These selected blocks were used to construct the phylogenetic trees separately using the maximum likelihood (ML) method implemented in RaxML (v8.2.4) (Stamatakis, 2014). The parameters were "raxmlHPC-PTHREADS-SSE3 -f a -N 1000 -m PROTGAMMALGX/GTRGAMMA -x 551314260 -p 551314260". Bootstrap analysis was performed with 1,000 replicates. The results showed that all nodes had bootstrap support of $100 \%$, indicating the reliability of phylogenetic recovery (Figure 4). In our phylogenetic trees, Cotinus and Pistacia were closely related. They belong to the tribe Rhoeae, together with Rhus and Toxicodendron. Unfortunately, few plastomes have been sequenced in Anacardiaceae, and we cannot describe the phylogenetic relationships of other Anacardiaceae plants in more detail based on plastome sequences. The application of NGS technology in Anacardiaceae plants is not sufficient; more plastome sequencing needs to be carried out in the future to improve the plastome resources of Anacardiaceae plants. 

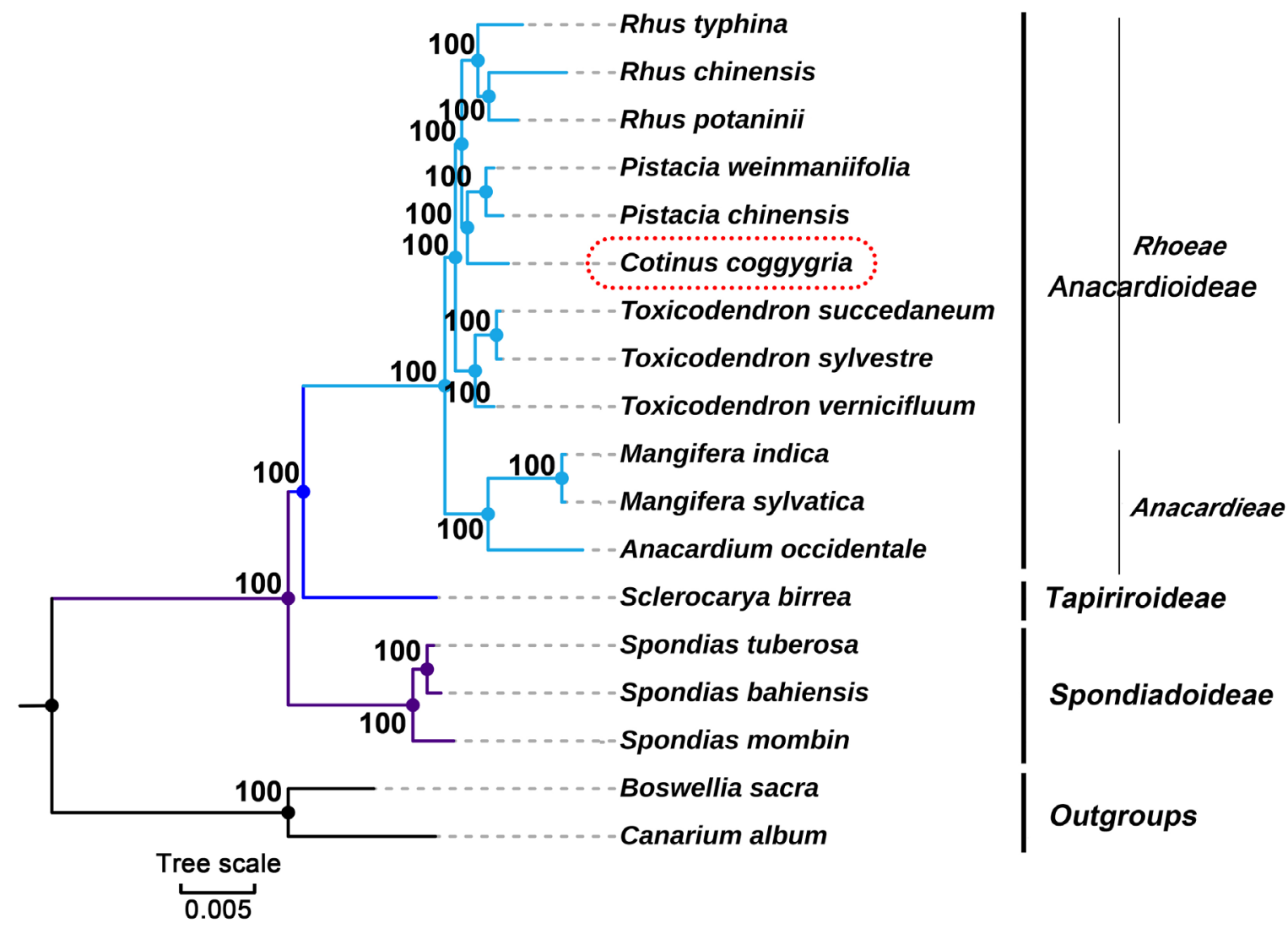

Figure 4- Phylogenetic relationships of species from Anacardiaceae family inferred using Maximum likelihood (ML) method. The phylogenetic tree was constructed by using the selected conserved blocks based on complete plastome sequences among the 18 plastomes. A total of 1,477 highly conservative blocks were identified by using Gblock with the default setting. Gblocks alignment (138,527 bp) accounts for $71 \%$ of the original alignment (194,522 bp). The number at the bottom of the scale, 0.005 , means that the length of the branch represents the replacement frequency of bases at each site of the genome at 0.005 . Bootstrap values were calculated from 1000 replicates. Two taxa from Burseraceae, namely, B. sacra and C. album were used as outgroups.

\section{Availability of data and materials}

The annotated plastome sequences and raw sequencing data of C. coggygria were deposited in GenBank (https://www. ncbi.nlm.nih.gov/) with the accession numbers MT876478 and SRR13076877. All the samples were deposited in the Herbarium of Southwest University, Chongqing, China. All other data and materials generated in this manuscript are available from the corresponding author upon reasonable request.

\section{Acknowledgments}

The authors thank Novogene (Tianjin) for technical support in DNA extraction and sequencing. This work was supported by the National Training Program of Innovation and Entrepreneurship for Undergraduates [X202010635443], National Natural Science Foundation of China [81260622], Chongqing Study Abroad Innovation Project [cx2019052], and the Science and Technology Research Program of Chongqing Education Commission of China [KJQN202001242].

\section{Conflicts of Interest}

The authors declare that there is no conflict of interest that could be perceived as prejudicial to the impartiality of the reported research.

\section{Author Contributions}

NZ and JY conceived the study, XYP and SXZ collected the samples and extracted DNA for next-generation sequencing, JLL assembled and annotated the complete plastid genome, JLL, XYP and LFX analyzed the plastid genome, and DQG wrote the manuscript. All authors have read and approved the final version.

\section{References}

Altschul SF, Gish W, Miller W, Myers EW and Lipman DJ (1990) Basic local alignment search tool. J Mol Biol 215:403-410.

Amiryousefi A, Hyvonen J and Poczai P (2018) IRscope: an online program to visualize the junction sites of chloroplast genomes. Bioinformatics 34:3030-3031.

Arseneau JR, Steeves R and Laflamme M (2017) Modified low-salt CTAB extraction of high-quality DNA from contaminant-rich tissues. Mol Ecol Resour 17:686-693.

Beier S, Thiel T, Munch T, Scholz U and Mascher M (2017) MISAweb: a web server for microsatellite prediction. Bioinformatics 33:2583-2585.

Bolger AM, Lohse M and Usadel B (2014) Trimmomatic: a flexible trimmer for Illumina sequence data. Bioinformatics 30:21142120.

Brudno M, Malde S, Poliakov A, Do CB, Couronne O, Dubchak I and Batzoglou S (2003) Glocal alignment: finding rearrangements during alignment. Bioinformatics 19:i54-i62. 
Castresana J (2000) Selection of conserved blocks from multiple alignments for their use in phylogenetic analysis. Mol Biol Evol 17:540-552.

Dierckxsens N, Mardulyn P and Smits G (2017) NOVOPlasty: de novo assembly of organelle genomes from whole genome data. Nucleic Acids Res 45:e18.

Emerman AB, Bowman SK, Barry A, Henig N, Patel KM, Gardner AF and Hendrickson CL (2017) NEBNext Direct: A novel, rapid, hybridization-based approach for the capture and library conversion of genomic regions of interest. Curr Protoc Mol Biol 119:7.30.31-37.30.24.

Frazer KA, Pachter L, Poliakov A, Rubin EM and Dubchak I (2004) VISTA: computational tools for comparative genomics. Nucleic Acids Res 32:W273-W279.

Gaudet P, Livstone MS, Lewis SE and Thomas PD (2011) Phylogenetic-based propagation of functional annotations within the Gene Ontology consortium. Brief Bioinform 12:449-462.

Greiner S, Lehwark P and Bock R (2019) OrganellarGenomeDRAW (OGDRAW) version 1.3.1: expanded toolkit for the graphical visualization of organellar genomes. Nucleic Acids Res 47:W59-W64.

Langmead B, Trapnell C, Pop M and Salzberg SL (2009) Ultrafast and memory-efficient alignment of short DNA sequences to the human genome. Genome Biol 10:R25.

Matić S, Stanić S, Mihailović M and Bogojević D (2016) Cotinus coggygria Scop.: an overview of its chemical constituents, pharmacological and toxicological potential. Saudi J Biol Sci 23:452-461.

Miao CY, Li Y, Yang J and Mao RL (2017) Landscape genomics reveal that ecological character determines adaptation: a case study in smoke tree (Cotinus coggygria Scop.). BMC Evol Biol 17:202.

Misra S and Harris N (2006) Using Apollo to browse and edit genome annotations. Curr Protoc Bioinformatics 12:9.5.1-9.5.28.

Rabah SO, Lee C, Hajrah NH, Makki RM, Alharby HF, Alhebshi AM, Sabir JSM, Jansen RK and Ruhlman TA (2017) Plastome sequencing of ten nonmodel crop species uncovers a large insertion of mitochondrial DNA in cashew. Plant Genome $10: 1-14$

Rozewicki J, Li S, Amada KM, Standley DM and Katoh K (2019) MAFFT-DASH: integrated protein sequence and structural alignment. Nucleic Acids Res 47:W5-W10.
Sánchez H, Fester T, Kloska S, Schröder W and Schuster W (1996) Transfer of rps19 to the nucleus involves the gain of an RNP-binding motif which may functionally replace RPS 13 in Arabidopsis mitochondria. EMBO J 15:2138-2149.

Shi L, Chen H, Jiang M, Wang L, Wu X, Huang L and Liu C (2019) CPGAVAS2, an integrated plastome sequence annotator and analyzer. Nucleic Acids Res 47:W65-W73.

Stamatakis A (2014) RAxML version 8: a tool for phylogenetic analysis and post-analysis of large phylogenies. Bioinformatics 30:1312-1313.

Sun SS, Fu PC, Zhou XJ, Cheng YW, Zhang FQ, Chen SL and Gao QB (2018) The complete plastome sequences of seven species in Gentiana sect. Kudoa (Gentianaceae): insights into plastid gene loss and molecular evolution. Front Plant Sci 9:493.

Talavera G and Castresana J (2007) Improvement of phylogenies after removing divergent and ambiguously aligned blocks from protein sequence alignments. Syst Biol 56:564-577.

Weglöhner W and Subramanian AR (1993) Nucleotide sequence of maize chloroplast rp132: completing the apparent set of plastid ribosomal protein genes and their tentative operon organization. Plant Mol Biol 21:543-548.

\section{Internet Resources}

GenBank, https://www.ncbi.nlm.nih.gov/genbank/ mVISTA Software, http://genome.lbl.gov/vista/mvista/submit.shtml IRscope Software, https://irscope.shinyapps.io/irapp/ MAFFT Software, https://mafft.cbrc.jp/alignment/software/ MISA, https://webblast.ipk-gatersleben.de/misa/

\section{Supplementary material}

The following online material is available for this article: Table S1 - Gene composition in the chloroplast genome of C. coggygria.

Table S2 - The Simple Sequence Repeats (SSRs) identified in the plastome of C. coggygria.

Table S3 - List of plastid genomes used for phylogenetic analysis.

Associate Editor: Rogério Margis

License information: This is an open-access article distributed under the terms of the Creative Commons Attribution License (type CC-BY), which permits unrestricted use, distribution and reproduction in any medium, provided the original article is properly cited. 